\title{
Modelling Spin relaxation in Semiconductor Quantum Wells: Modifying the Elliot Process
}

\author{
M.P. Vaughan and J.M. Rorison \\ University of Bristol, Bristol BS8 1TR, United Kingdom \\ E-mail: martinpaul.vaughan@physics.org
}

\begin{abstract}
A model of the Elliot process for spin relaxation is developed that explicitly incorporates the Dresselhaus spin-splitting of the conduction band in semiconductors lacking an inversion symmetry. It is found that this model reduces to existing models in bulk if the scattering matrices are constructed from a superposition of eigenstates. It is shown that the amplitude for intra-subband spin relaxation disappears in quantum wells on the basis of existing models. However, an amplitude due to the Dresselhaus spin-splitting remains, becoming increasingly important as the well becomes narrower. It is also shown that this component does not disappear for scattering between spin states at the same wavevector. It is concluded that for quantum wells and lower dimensional semiconductors that this modified model should be used in analysis of the spin dynamics.
\end{abstract}

PACS numbers: 71.70.Ej, 72.25.Rb, 73.21.-b

Keywords: spin orbit interaction, spin relaxation, quantum wells

Submitted to: Semicond. Sci. Technol. 


\section{Introduction}

The manipulation of electron spin in semiconductor materials is key for applications in both 'classical' photonic devices, such as spin vertical cavity surface emitting lasers, where the spin can be used as an additional label in optical communications, or in quantum information processing devices where the electron spin is manipulated to process the information. This requires the understanding of the physics of electron spin to be reconsidered, particularly when in systems of high confinement such as quantum wells and quantum dots. The electron spin is strongly coupled to the orbital properties of the material so material confinement could be expected to strongly effect spin relaxation. The aim of this paper is to examine this timely issue and develop a formalism for future use for these applications.

The spin orbit interaction (SOI) is well-known to play an important role in spin relaxation mechanisms. This is a consequence of its effect on the band structure of a material. Not only does spin-orbit coupling split the degeneracy of the valence bands but it modifies the spin properties of conduction band electrons in two important ways. Firstly, the electronic states are now eigenvectors of the total angular momentum $\mathbf{J}$, rather that orbital angular momentum $\mathbf{L}$ or spin $\mathbf{S}$ alone, meaning that they exist as an admixture of pure spin states [1]. Secondly, in materials lacking an inversion symmetry, for non-zero k-vector, the degeneracy of the conduction band is split by an energy proportion to the cube of the $\mathbf{k}$-vector magnitude [2].

The importance of the first of these processes for spin relaxation was first pointed out by Elliot [1], who considered materials with an inversion symmetry, such as Si and Ge with the diamond structure. Since the eigenvectors exist as an admixture of states, there will be a finite amplitude for scattering between opposite spin states, even when

the scattering potential is non-magnetic (i.e. it will not flip spin directly). This is known as the Elliot process, although very often in the literature it is lumped together with the Yafet process [3], which is due to the modulation of the spin-orbit interaction by phonons. The two processes together are then refered to as the Elliot-Yafet (EY) mechanism. We do not consider the Yafet process in the present work.

The Elliot process has subsequently been considered for materials lacking an inversion symmetry, notably by Zawadzki and Szymańska [4] and Chazalviel [5]. We shall refer to these later treatments collectively as the ZSC model for ease of reference. Although widely used and accepted as a practical implementation of the Elliot process, this ZSC model does not incorporate the spin-splitting of the bands.

The splitting of the conduction band, first reported and named after Dresselhaus [2], is formally analogous to the Zeeman splitting of energy levels by a magnetic field and may be modeled in a two-band model accordingly. The solutions of this model are the spin eigenstates of an effective magnetic field, with a $\mathbf{k}$-dependent direction. In this case, most non-magnetic scattering events will not flip the spin, but will scatter the electron to a new $\mathbf{k}$ state in which the spin sees a different effective magnetic field and is projected into a superposition of new spin eigenstates. The spin then 
precesses around this effective field direction. Further scattering processes progressively lose memory of any initial spin polarization in a sequence of random walks. This is the basis of the important D'yakonov-Perel (DP) mechanism for spin relaxation [6]. At room temperature, the DP mechanism has been shown by Song and Kim [7] to be the dominant mechanism of spin relaxation in bulk $n$-type semiconductors. At lower temperatures, where quantum informatic applications may be more relevant, these authors indicate that the Elliot-Yafet mechanism becomes more important. Early calculations for bulk GaAs by Fishman and Lampel [8] suggested that the energy dependent spin relaxation time $\tau_{s}(\epsilon)$ will be around 100 ns for both DP and EY processes at an electronic energy of $\epsilon=10 \mathrm{meV}$, decreasing to around $6.7 \mathrm{~ns}$ and $1.7 \mathrm{~ns}$ respectively at the room temperature thermal energy of $\epsilon=39 \mathrm{meV}$. Experimental studies for GaAs quantum wells [9-11], however, report spin relaxation times on the order 10 - 100 ps at room temperature.

The aim of this paper is to treat the physics of these mechanisms consistently by incorporating the Dresselhaus splitting of the conduction band into the formalism of the Elliot process. Specifically, we will find that the existing ZSC model is adequate to treat spin relaxation in bulk material but fails for low dimensional systems, such as quantum wells, where the introduction of the Dresselhaus terms becomes increasingly important as the quantum confinement is increased.

Our principal objective will be to derive explicit expressions for the coupling factor $\left\langle\phi_{\mathbf{k}^{\prime} \downarrow} \mid \phi_{\mathbf{k} \uparrow}\right\rangle$ between states of opposite spin in both bulk and quantum wells when the Dresselhaus spin-orbit splitting is incorporated. We may then use this to (i) obtain an estimation of the spin relaxation time in terms of elastic momentum relaxation times for the associated non-magnetic scattering potential and (ii) provide a theoretical framework for developing more detailed models involving, for instance, non-isotropic scattering potentials or inelastic scattering via polar optical phonons.

A general description of the Elliot process is given in Section 2, where we describe, in general, how spin relaxation may arise via a non-magnetic scattering potential when the states of the system are an admixture of pure spin states. There, we show how, for elastic scattering by an isotropic scattering potential, the spin relaxation time may be expressed in terms of the momentum relaxation time associated with the scattering potential. For elastic scattering, this expression remains a useful approximation even for non-isotropic scattering potentials and so may be used to investigate the relative importance of the Dresselhaus contribution to spin relaxation.

In Section 3, we consider how the ZSC model may be modified, starting with a description of the Dresselhaus SOI in Section 3.1. We then give explicit results for the modified model for the bulk case (the details of the derivation being relegated to the Appendix). By representing the spin as a superposition of the spin eigenstates of the system, it is then shown in Section 3.3 that the ZSC model remains valid for the bulk case with only a negligible term (referred to as the Dresselhaus coupling factor) arising from the Dresselhaus SOI.

Turning our attention to symmetric quantum wells in Section 3.4, we find that the amplitude for intra-subband scattering disappears in the ZSC model. However, the 
Dresselhaus coupling factor remains, becoming increasingly important as the quantum well becomes narrower. We will show that it is comparable to the ZSC coupling factor in bulk for GaAs wells of around $4 \mathrm{~nm}$ wide and exceeding it for narrower wells. Hence, we conclude in Section 4 that this modified version of the Elliot process should be used for the analysis of spin dynamics in quantum wells and lower dimensional semiconductors.

Finally, in the Appendices, we include both a derivation of Dresselhaus spinsplitting and the admixture eigenstates using $\mathbf{k} \cdot \mathbf{p}$ theory for the benefit of the interested reader, as well as some of the mathematical details involved in the derivation of the coupling factors.

\section{The Elliot process of spin relaxation}

As a consequence of the spin-orbit interaction (see Appendix A.1), the periodic part of the conduction band Bloch state in semiconductor materials becomes an admixture of pure spin states, which, for instance, may be expressed as

$$
\left|\phi_{\mathbf{k} \uparrow}\right\rangle=\left|a_{\mathbf{k} \uparrow}\right\rangle|\uparrow\rangle+\left|b_{\mathbf{k} \uparrow}\right\rangle|\downarrow\rangle,
$$

where the $\uparrow$ in the subscript labels the major component of the spin. If we consider the scattering of a Bloch function $\left|\psi_{\mathbf{k} \uparrow}\right\rangle=e^{i \mathbf{k} \cdot \mathbf{r}}\left|\phi_{\mathbf{k} \uparrow}\right\rangle$ to a state $\left|\psi_{\mathbf{k}^{\prime} \downarrow}\right\rangle$ via a non-magnetic potential $V$ (i.e. which does not flip spin), we have

$$
\left\langle\psi_{\mathbf{k}^{\prime} \downarrow}|V| \psi_{\mathbf{k} \uparrow}\right\rangle=V_{\mathbf{k}-\mathbf{k}^{\prime}}\left\langle\phi_{\mathbf{k}^{\prime} \downarrow} \mid \phi_{\mathbf{k} \uparrow}\right\rangle
$$

and

$$
\left\langle\phi_{\mathbf{k}^{\prime} \downarrow} \mid \phi_{\mathbf{k} \uparrow}\right\rangle=\left\langle a_{\mathbf{k}^{\prime} \downarrow} \mid b_{\mathbf{k} \uparrow}\right\rangle+\left\langle b_{\mathbf{k}^{\prime} \downarrow} \mid a_{\mathbf{k} \uparrow}\right\rangle,
$$

where we have assumed that the potential $V$ is slowly varying over the scale of primitive cell and factorized out the integral $V_{\mathbf{k}-\mathbf{k}^{\prime}}=\left\langle e^{-\mathbf{i} \mathbf{k}^{\prime} \cdot \mathbf{r}}|V| e^{\mathrm{ik} \cdot \mathbf{r}}\right\rangle$. Hence, we will have a finite amplitude to flip the spin even though $V$ does not flip spin directly.

For elastic processes, we may define a spin-flipping rate using Fermi's Golden rule as

$$
W_{\mathbf{k}^{\prime} \mathbf{k}}^{\downarrow \uparrow}=\frac{2 \pi}{\hbar}\left|V_{\mathbf{k}-\mathbf{k}^{\prime}}\right|^{2}\left|\left\langle\phi_{\mathbf{k}^{\prime} \downarrow} \mid \phi_{\mathbf{k} \uparrow}\right\rangle\right|^{2} \delta\left(\epsilon_{\mathbf{k}^{\prime}}-\epsilon_{\mathbf{k}}\right) .
$$

Integrating this over $\mathbf{k}^{\prime}$, we obtain a total spin-flipping rate $W_{\mathbf{k}}^{\downarrow \uparrow}$ for the state $\left|\phi_{\mathbf{k} \uparrow}\right\rangle$. At this point, the mathematics is simplified by assuming that $V_{\mathbf{k}-\mathbf{k}^{\prime}}$ is isotropic, which is true in the important case of deformation potential acoustic phonon scattering. Due to the action of the delta function, $W_{\mathbf{k}}^{\downarrow \uparrow}$ is effectively averaged over the angular direction of $\mathbf{k}^{\prime}$. Similarly, we may integrate over the angular direction of $\mathbf{k}$ to obtain an energy dependent rate, or in terms of a relaxation time $\tau_{s}$,

$$
\frac{1}{\tau_{s}\left(\epsilon_{\mathbf{k}}\right)}=\frac{2 \pi}{\hbar}\left|V_{\mathbf{k}-\mathbf{k}^{\prime}}\right|^{2}\left\langle\left\langle\left|\left\langle\phi_{\mathbf{k}^{\prime} \downarrow} \mid \phi_{\mathbf{k} \uparrow}\right\rangle\right|^{2}\right\rangle\right\rangle \rho\left(\epsilon_{\mathbf{k}}\right),
$$

where the outer angled brackets imply double integration over the angular directions of $\mathbf{k}^{\prime}$ and $\mathbf{k}$ and $\rho\left(\epsilon_{\mathbf{k}}\right)$ is the density of states. 
Meanwhile, the spin-conserving transition gives the momentum relaxation time

$$
\frac{1}{\tau_{m}(\mathbf{k})}=\frac{2 \pi}{\hbar} \sum_{\mathbf{k}^{\prime}}\left|V_{\mathbf{k}-\mathbf{k}^{\prime}}\right|^{2}\left|\left\langle\phi_{\mathbf{k}^{\prime} \uparrow} \mid \phi_{\mathbf{k} \uparrow}\right\rangle\right|^{2}(1-\cos \alpha) \delta\left(\epsilon_{\mathbf{k}^{\prime}}-\epsilon_{\mathbf{k}}\right),
$$

where $\alpha$ is the angle between $\mathbf{k}$ and $\mathbf{k}^{\prime}$. The cosine term emerges from the solution to Boltzmann's transport equation for a low electric field for elastic scattering. Typically, the factor $\left|\left\langle\phi_{\mathbf{k}^{\prime} \uparrow} \mid \phi_{\mathbf{k} \uparrow}\right\rangle\right|^{2}$ is taken to be approximately equal to unity for small $k$-vectors where the energy bands may be taken to be parabolic, so, making the same assumptions as above and integrating over the direction of $\mathbf{k}$, we have

$$
\frac{1}{\tau_{m}\left(\epsilon_{\mathbf{k}}\right)}=\frac{2 \pi}{\hbar}\left|V_{\mathbf{k}-\mathbf{k}^{\prime}}\right|^{2}\left\langle\left\langle\left|\left\langle\phi_{\mathbf{k}^{\prime} \uparrow} \mid \phi_{\mathbf{k} \uparrow}\right\rangle\right|^{2}\right\rangle\right\rangle \rho\left(\epsilon_{\mathbf{k}}\right)
$$

For non-isotropic processes, such as piezoelectric acoustic phonon scattering, $V_{\mathbf{k}-\mathbf{k}^{\prime}}$ may contain terms in $\cos \alpha$, so the exact expressions will differ from that derived above. They will, however, remain on the same order of magnitude, which suffices for this present investigation. Combining (5) and (7), we may directly compare the energy dependent spin and momentum relaxation times via

$$
\frac{\tau_{m}(\epsilon)}{\tau_{s}(\epsilon)}=\frac{\left\langle\left\langle\left|\left\langle\phi_{\mathbf{k}^{\prime} \downarrow} \mid \phi_{\mathbf{k} \uparrow}\right\rangle\right|^{2}\right\rangle\right\rangle}{\left\langle\left\langle\left|\left\langle\phi_{\mathbf{k}^{\prime} \uparrow} \mid \phi_{\mathbf{k} \uparrow}\right\rangle\right|^{2}\right\rangle\right\rangle} .
$$

Typically, it is found that $\tau_{m} / \tau_{s} \ll 1$.

Explicit forms for (1) giving the admixture components of the conduction band state have been found by Zawadzki and Szymańska [4] and Chazalviel [5] on the basis of the Kane model [12], which we shall refer to as the ZSC model. However, these expressions did not incorporate the Dresselhaus splitting of the conduction band into

k-dependent spin states in materials lacking an inversion symmetry. This splitting acts like an effective magnetic field and leads to spin relaxation via the D'yakonovPerel mechanism [6], which is often the principal spin relaxation process in bulk and 2D materials. This spin-splitting and its incorporation into the factor $\left\langle\phi_{\mathbf{k}^{\prime} \downarrow} \mid \phi_{\mathbf{k} \uparrow}\right\rangle$ is discussed in the next section.

\section{Modified Elliot process}

\subsection{Spin-splitting of the conduction band}

In the ZSC model, the overlap factor $\left\langle\phi_{\mathbf{k}^{\prime} \downarrow} \mid \phi_{\mathbf{k} \uparrow}\right\rangle$ is found using fixed spin-states for all $\mathbf{k}$ states. However, this does not take into account the $\mathbf{k}$-dependence of the spin direction associated with the Dresselhaus spin-splitting of the conduction band, which arises due to the spin-orbit interaction in materials lacking an inversion symmetry (a full derivation is given in Appendix B). The Dresselhaus spin-orbit interaction is often presented in terms of a two band model of the form

$$
H=H_{0}+H_{D}
$$


where $H_{0}$ gives the $k^{2}$ dependent part of the energy and the conduction band splitting is determined by the Hamiltonian

$$
H_{D}=\gamma_{D}(\boldsymbol{\sigma} \cdot \boldsymbol{\kappa}) \text {. }
$$

Here $\gamma_{D}$ is the Dresselhaus coefficient and $\boldsymbol{\kappa}$ is a vector with components

$$
\kappa_{z}=k_{z}\left(k_{x}^{2}-k_{y}^{2}\right)
$$

and cyclic permutations thereof (expressions for $\gamma_{D}$ in terms of band parameters are given in Appendix A.2 and derived from $\mathbf{k} \cdot \mathbf{p}$ theory in Appendix B). The Hamiltonian may be written explicitly in matrix form as

$$
H_{D}=\gamma_{D}\left[\begin{array}{cc}
\kappa_{z} & \kappa_{x}-\mathrm{i} \kappa_{y} \\
\kappa_{x}+\mathrm{i} \kappa_{y} & -\kappa_{z}
\end{array}\right]
$$

The eigenvalues of (12) are

$$
\pm \epsilon_{D}(\mathbf{k})= \pm \gamma_{D}\left(\kappa_{x}^{2}+\kappa_{y}^{2}+\kappa_{z}^{2}\right)^{1 / 2}
$$

with eigenvectors

$$
\left|\chi_{\mathbf{k}}^{+}\right\rangle=\left[\begin{array}{c}
\cos (\theta / 2) e^{-\mathrm{i} \phi} \\
\sin (\theta / 2)
\end{array}\right],\left|\chi_{\mathbf{k}}^{-}\right\rangle=\left[\begin{array}{c}
-\sin (\theta / 2) e^{-\mathrm{i} \phi} \\
\cos (\theta / 2)
\end{array}\right]
$$

Here, $\theta$ and $\phi$ are the spherical polar coordinates of the vector $\boldsymbol{\kappa}$ (not to be confused with the coordinates of $\mathbf{k}$ ). Hence, we that the spin states are $\mathbf{k}$-dependent.

From inspection of (11) we see that if $\mathbf{k}=(k, 0,0)$ or $k_{x}=k_{y}=k_{z}$, then all the components $\kappa_{i}=0$ and hence, from (13), the spin-splitting is zero along the [100] and [111] directions. Along the [110] direction we have $\mathbf{k}=(k, k, 0) / \sqrt{2}$ and we find $\epsilon_{D}=\gamma_{D} k^{3} / 2$, which may be shown to be a local maximum. Using parameters for GaAs of $\gamma_{D}=18 \mathrm{eVA}^{3}$ [13] and $m^{*} / m_{0}=0.067$ [14], we find for $\epsilon_{k}=\hbar^{2} k^{2} /\left(2 m^{*}\right)=0.4 \mathrm{eV}$ along the [110] direction (roughly 10 times the thermal energy $3 k_{B} T / 2$ at $300 \mathrm{~K}$ ), $\epsilon_{D}$ is around $5 \mathrm{meV}$.

\subsection{Modified eigenvectors}

Incorporating the $\mathbf{k}$-dependent spin-splitting of the conduction band, it can be shown (see Appendix C) that the general form of the periodic part of the conduction band states in bulk material may be written as

$$
\left|u_{\mathbf{k}}^{ \pm}\right\rangle=U_{\mathbf{k}}^{ \pm}\left|\chi_{\mathbf{k}}^{ \pm}\right\rangle
$$

where $U_{\mathbf{k}}^{ \pm}$is an operator of the form

$$
U_{\mathbf{k}}^{ \pm}=\Gamma_{\mathbf{k}}^{ \pm} U_{\mathbf{k}}
$$

and 


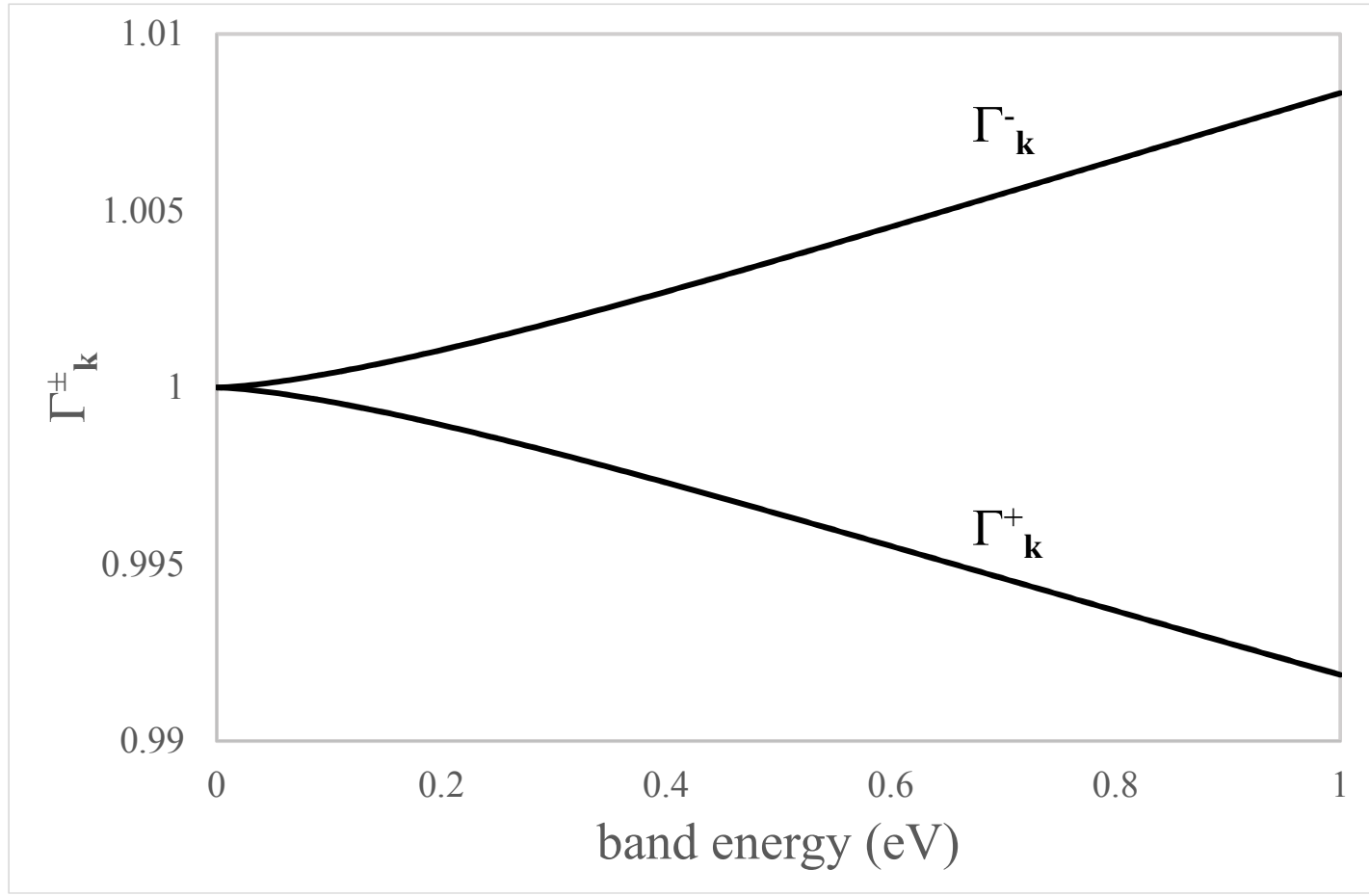

Figure 1. Variation of the dimensionless spin-dependent factor $\Gamma_{\mathbf{k}}^{ \pm}$of (16) multiplying the conduction band eigenstates for GaAs as a function of band energy along the [110] direction. Here we have used $\gamma_{D}=18 \mathrm{eV}^{3}$ [13] to calculate the spin-splitting. As seen from the scale, this factor is very close to unity for spin, as required for the validity of the ZSC model in bulk as described in the text.

$$
U_{\mathbf{k}}=\left[a|\mathrm{i} S\rangle+c \mathbf{K}^{*} \cdot|\mathbf{R}\rangle+\frac{\mathrm{i} b}{\sqrt{2}}\left(\mathbf{K}^{*} \times|\mathbf{R}\rangle\right) \cdot \boldsymbol{\sigma}\right] .
$$

Here we have defined a vector of states via

$$
|\mathbf{R}\rangle \equiv \mathbf{e}_{x}|X\rangle+\mathbf{e}_{y}|Y\rangle+\mathbf{e}_{z}|Z\rangle
$$

and

$$
\mathbf{K}=\mathbf{e}_{x} K_{x}+\mathbf{e}_{y} K_{y}+\mathbf{e}_{z} K_{z}
$$

where the $\mathbf{e}_{i}$ are unit vectors in the Cartesian directions and the $K_{i}$ are $\mathbf{k}$-dependent terms defined in (A.7). These terms include the momentum matrix describing the coupling of the conduction band with the valence bands, as well as the coupling with higher conduction bands that gives the Dresselhaus spin splitting. The coefficients in (17) are given in terms of the band gap $\epsilon_{G}$ and spin split-off energy $\Delta$ by

$$
a=\frac{\epsilon_{G}\left(\epsilon_{G}+\Delta\right)}{D}
$$




$$
b=\frac{\sqrt{2} \Delta}{3 D}
$$

and

$$
c=\frac{\left(\epsilon_{G}+2 \Delta / 3\right)}{D},
$$

where

$$
\begin{aligned}
D & =\left(\epsilon_{G}^{2}\left(\epsilon_{G}+\Delta\right)^{2}\right. \\
& \left.+\left[(2 / 3)\left(\epsilon_{G}+\Delta\right)^{2}+\epsilon_{G}^{2} / 3\right] P^{2} k^{2}\right)^{1 / 2}
\end{aligned}
$$

and $P$ is the Kane momentum matrix defined by (A.3). Lastly, the spin-dependent factor $\Gamma_{\mathbf{k}}^{ \pm}$is given by

$$
\Gamma_{\mathbf{k}}^{ \pm}=\sqrt{\frac{D^{2}}{D^{2} \pm V_{\mathbf{k}}}}
$$

where

$$
V_{\mathbf{k}}=\epsilon_{G}\left(\epsilon_{G}+\Delta\right)\left(2 \epsilon_{G}+\Delta\right) \epsilon_{D}(\mathbf{k}) .
$$

The factor $\Gamma_{\mathbf{k}}^{ \pm}$remains very close to unity varying only by $1 \%$ as the band energy reaches $1 \mathrm{eV}$ along the [110] direction, in which direction the spin-splitting is a maximum (see Fig. 1).

\subsection{Spin relaxation}

Inspecting the form of (17), we see that that a general eigenvector is, in fact, an admixture of pure spin states, brought about through the action of $\left(\mathbf{K}^{*} \times|\mathbf{R}\rangle\right) \cdot \boldsymbol{\sigma}$ on a spinor. This component of opposite spin is small so, on scattering from one $\mathbf{k}$ state to another by a non-magnetic potential, spin-conserving transitions will be more likely than spin-flipping ones. However, since the spin components of a state are $\mathbf{k}$ dependent, the spin eigenvectors of the final state will be different. Hence, for a spin-conserving transtion, the spin will be projected onto a different set of basis states. Since the spin is now in a superposition of the spin eigenstates of the system (which are themselves admixtures of pure spin states), it will precess with a Larmor frequency $\Omega_{\mathbf{k}}=2 \gamma_{D}|\boldsymbol{\kappa}| / \hbar$. The vector $\boldsymbol{\Omega}_{\mathbf{k}}$ then acts as an effective magnetic field.

Subsequent spin-conserving scattering events randomize the component of spin in any given direction by randomizing the direction $\boldsymbol{\Omega}_{\mathbf{k}}$. This is then spin relaxation via the D'yakonov-Perel mechanism [6], which is a 'motional narrowing' process. That is, greater interaction with the system gives less time for the spin to precess and so the spin relaxation time is inversely proportional to the momentum relaxation time. 
In the ZSC model, spin relaxation is due to spin-flipping transitions via coupling of the opposite spin-components according to (2) and (3). This form, however, is for transitions between eigenstates of $\sigma_{z}$; namely

$$
|\uparrow\rangle=\left[\begin{array}{l}
1 \\
0
\end{array}\right] \text { and }|\downarrow\rangle=\left[\begin{array}{l}
0 \\
1
\end{array}\right] \text {. }
$$

It does not allow for the k-dependence of the spin states. However, as discussed above, the electron is generally in a superposition of spin states. Otherwise there would be no D'yakonov-Perel process for spin relaxtion. Let us consider such a superposition of eigenstates having a principal component of spin $|\uparrow\rangle$. The principle spin component of an eigenvector $\left|u_{\mathbf{k}}^{ \pm}\right\rangle$is $\left|\chi_{\mathbf{k}}^{ \pm}\right\rangle$, although, as argued above, it will have a small component of $\left|\chi_{\mathbf{k}}^{\mp}\right\rangle$. Now, $|\uparrow\rangle$ may be projected onto to the $\left|\chi_{\mathbf{k}}^{ \pm}\right\rangle$states as

$$
|\uparrow\rangle=\left|\chi_{\mathbf{k}}^{+}\right\rangle\left\langle\chi_{\mathbf{k}}^{+} \mid \uparrow\right\rangle+\left|\chi_{\mathbf{k}}^{-}\right\rangle\left\langle\chi_{\mathbf{k}}^{-} \mid \uparrow\right\rangle
$$

so the superposition of eigenstates with this principle component of spin will be weighted in the same proportion as

$$
\left|\phi_{\mathbf{k} \uparrow}\right\rangle=\left|u_{\mathbf{k}}^{+}\right\rangle\left\langle\chi_{\mathbf{k}}^{+} \mid \uparrow\right\rangle+\left|u_{\mathbf{k}}^{-}\right\rangle\left\langle\chi_{\mathbf{k}}^{-} \mid \uparrow\right\rangle .
$$

Let us write this in the form of (1) as

$$
\left|\phi_{\mathbf{k} \uparrow}\right\rangle=\left|a_{\mathbf{k} \uparrow}\right\rangle|\uparrow\rangle+\left|b_{\mathbf{k} \uparrow}\right\rangle|\downarrow\rangle,
$$

where $\left|a_{\mathbf{k} \uparrow}\right\rangle=\left\langle\uparrow \mid \phi_{\mathbf{k} \uparrow}\right\rangle$ and $\left|b_{\mathbf{k} \uparrow}\right\rangle=\left\langle\downarrow \mid \phi_{\mathbf{k} \uparrow}\right\rangle$. Using (15), we have, from (28),

$$
\left\langle\uparrow \mid \phi_{\mathbf{k} \uparrow}\right\rangle=\left\langle\uparrow\left|U_{\mathbf{k}}^{+}\right| \chi_{\mathbf{k}}^{+}\right\rangle\left\langle\chi_{\mathbf{k}}^{+} \mid \uparrow\right\rangle+\left\langle\uparrow\left|U_{\mathbf{k}}^{-}\right| \chi_{\mathbf{k}}^{-}\right\rangle\left\langle\chi_{\mathbf{k}}^{-} \mid \uparrow\right\rangle
$$

Now, if $U_{\mathrm{k}}^{+} \approx U_{\mathrm{k}}^{-}=U_{\mathrm{k}}$, implying $\Gamma_{\mathrm{k}}^{+} \approx \Gamma_{\mathrm{k}}^{-} \approx 1$, this reduces to

$$
\left|a_{\mathbf{k} \uparrow}\right\rangle=\left\langle\uparrow \mid \phi_{\mathbf{k} \uparrow}\right\rangle=\left\langle\uparrow\left|U_{\mathbf{k}}\right| \uparrow\right\rangle
$$

(since $\sum_{\alpha}\left|\chi_{\mathbf{k}}^{\alpha}\right\rangle\left\langle\chi_{\mathbf{k}}^{\alpha}\right|=1$ ) and similarly

$$
\left|b_{\mathbf{k} \uparrow}\right\rangle=\left\langle\downarrow \mid \phi_{\mathbf{k} \uparrow}\right\rangle=\left\langle\downarrow\left|U_{\mathbf{k}}\right| \uparrow\right\rangle \text {. }
$$

As shown in Fig. $1, \Gamma_{\mathbf{k}}^{ \pm} \approx 1$ holds as a very good approximation over a large energy range. Hence, we can rewrite (29) as

$$
\left|\phi_{\mathbf{k} \uparrow}\right\rangle=|\uparrow\rangle\left\langle\uparrow\left|U_{\mathbf{k}}\right| \uparrow\right\rangle+|\downarrow\rangle\left\langle\downarrow\left|U_{\mathbf{k}}\right| \uparrow\right\rangle,
$$

which does not explicitly depend on the underlying spin eigenstates. In this case, we can simply use the prescription implied by (2) and (3) to obtain the spin-flipping amplitude for a fixed spin orientation $|\uparrow\rangle$, without concern for the $\mathbf{k}$-dependence of the spin states.

The coupling factor may then be simplified to be

$$
\left\langle\phi_{\mathbf{k}^{\prime} \downarrow} \mid \phi_{\mathbf{k} \uparrow}\right\rangle=\left\langle\downarrow\left|U_{\mathbf{k}^{\prime}}^{\dagger} U_{\mathbf{k}}\right| \uparrow\right\rangle,
$$

where the $\dagger$ superscript indicates complex conjugation. Note that had we not had $\Gamma_{\mathbf{k}}^{ \pm} \approx 1$, using (28), this expression and the squared modulus of it would have contained 


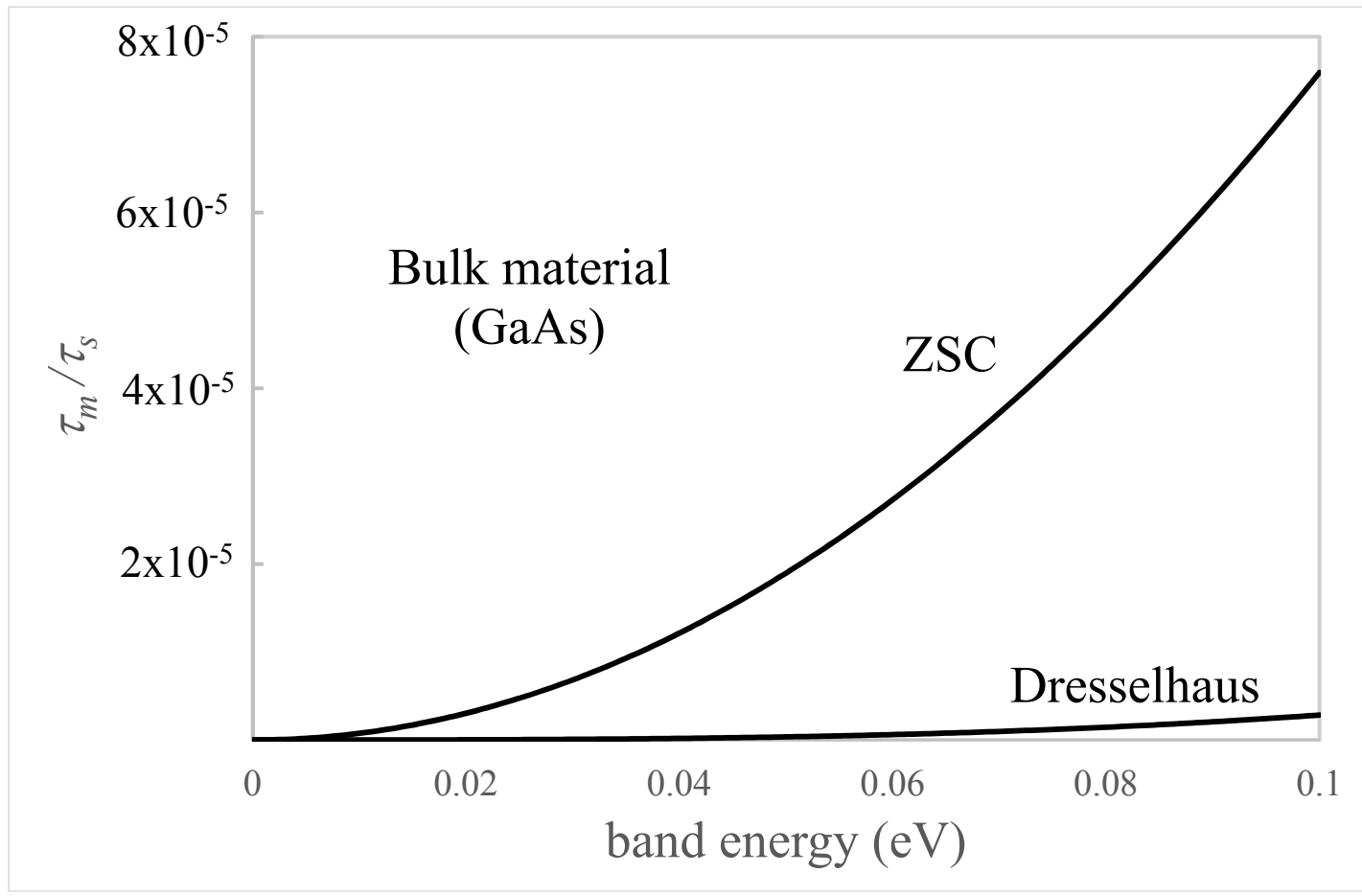

Figure 2. The squared moduli of the coupling factors $\tau_{m} / \tau_{s} \approx\left|\left\langle\phi_{\mathbf{k}^{\prime} \downarrow} \mid \phi_{\mathbf{k} \uparrow}\right\rangle\right|^{2}$ for the ZSC and Dresselhaus components in bulk material. Both give a ratio of the spin relaxation rate to the momentum relaxation rate (here, the averaging over angular direction is approximated as introducing a factor of unity). As can be seen, in bulk the Dresselhuas contribution is negligible and the ZSC description remains valid.

4 and 8 terms respectively, so (34) represents a significant simplification. Using (17) and neglecting terms in $k^{3}$ and higher, we show in Appendix D that (34) becomes

$$
\left\langle\phi_{\mathbf{k}^{\prime} \downarrow} \mid \phi_{\mathbf{k} \uparrow}\right\rangle=-\frac{\epsilon_{k}}{\epsilon_{G}^{*}} \frac{k^{\prime}}{k}\left[\sin \vartheta^{\prime} \cos \vartheta e^{\mathrm{i} \varphi^{\prime}}-\sin \vartheta \cos \vartheta^{\prime} e^{\mathrm{i} \varphi}\right],
$$

where $\epsilon_{k}=\hbar^{2} k^{2} /\left(2 m^{*}\right)$ and

$$
\frac{1}{\epsilon_{G}^{*}}=\left(1-\frac{m^{*}}{m_{0}}\right) \frac{\Delta\left(\Delta+2 \epsilon_{G}\right)}{\epsilon_{G}\left(\epsilon_{G}+\Delta\right)\left(3 \epsilon_{G}+2 \Delta\right)},
$$

which is the result of the ZSC model. Here $\vartheta$ and $\varphi$ are the spherical polar coordinates of $\mathbf{k}$-vector. Note that if $\mathbf{k}^{\prime}=\mathbf{k}$, the coupling factor is zero in this model.

We note that, from (D.6) we see that there is still a small residual coupling when $\mathbf{k}^{\prime}=\mathbf{k}$ proportional to the Dresselhaus coefficient, given by

$$
\left\langle\phi_{\mathbf{k} \downarrow} \mid \phi_{\mathbf{k} \uparrow}\right\rangle=\gamma_{D} \frac{\left(\Delta+2 \epsilon_{G}\right)}{\epsilon_{G}\left(\epsilon_{G}+\Delta\right)}\left(\kappa_{x}+\mathrm{i} \kappa_{y}\right) .
$$

Although small, this expression is only precisely zero in the [001] and [111] directions, when the conduction band spin-splitting is zero. However, as we shall see, even the 
maximum value of this contribution is negligible compared to the ZSC component of (35) confirming that the ZSC model remains and adequate description of the Elliot process in bulk material. The only caveat is that we must regard the spin states $|\uparrow\rangle$ and $|\downarrow\rangle$ as superpositions of the spin eigenstates of the system.

For the purposes of comparing the resultant spin relaxation times arising out of (35) and (37) to the momentum relaxation time via (8), we note that

$$
\left\langle\phi_{\mathbf{k}^{\prime} \uparrow} \mid \phi_{\mathbf{k} \uparrow}\right\rangle=\left\langle a_{\mathbf{k}^{\prime} \uparrow} \mid a_{\mathbf{k} \uparrow}\right\rangle+\left\langle b_{\mathbf{k}^{\prime} \uparrow} \mid b_{\mathbf{k} \uparrow}\right\rangle \approx\left\langle a_{\mathbf{k}^{\prime} \uparrow} \mid a_{\mathbf{k} \uparrow}\right\rangle,
$$

for small $k$. In the same approximation, $\left\langle a_{\mathbf{k}^{\prime} \uparrow} \mid a_{\mathbf{k} \uparrow}\right\rangle \approx a^{2}$, which from (20) using $D^{2} \approx \epsilon_{G}^{2}\left(\epsilon_{G}+\Delta\right)^{2}$ is approximately equal to unity. Hence, we may consider (35) and (37) to be normalised and use

$$
\frac{\tau_{m}}{\tau_{s}} \approx\left\langle\left\langle\left|\left\langle\phi_{\mathbf{k}^{\prime} \downarrow} \mid \phi_{\mathbf{k} \uparrow}\right\rangle\right|^{2}\right\rangle\right\rangle .
$$

The ZSC and Dresselhaus components are plotted for GaAs in Fig. 2. Here, we have not explicitly carried out the averaging over angular direction, since this will only introduce a factor on the order of unity and we only interested in order of magnitude estimates. In both cases we assume elastic scattering processes, so in (35) we put $k^{\prime}=k$ and plot the maximum value of $\left|\left\langle\phi_{\mathbf{k}^{\prime} \downarrow} \mid \phi_{\mathbf{k} \uparrow}\right\rangle\right|^{2}=\left(\epsilon_{k} / \epsilon_{G}^{*}\right)^{2}$ as a function of energy. For the Dresselhaus component of (37) we put the $\mathbf{k}$-dependent factor to $k^{3}$ for the sake of comparison, so that $\left|\left\langle\phi_{\mathbf{k}^{\prime} \downarrow} \mid \phi_{\mathbf{k} \uparrow}\right\rangle\right|^{2}$ varies as $k^{6}$.

We note that both components are small even at high energy, implying that the spin-relaxation rate is much smaller than the momentum relaxation rate. At $\epsilon_{k}=$ $0.04 \mathrm{eV}$ (the thermal energy $3 k_{B} T / 2$ at $300 \mathrm{~K}$ ) a momentum relaxation time of $1 \mathrm{ps}$ would give a spin relaxation time of around $90 \mathrm{~ns}$ in the ZSC model. At this energy, the Dresselhaus component is much smaller, being about $1 \%$ of the ZSC component, rising to about $4 \%$ at $\epsilon_{k}=0.1 \mathrm{eV}$, so we may consider this to be negligible.

\subsection{Quantum wells}

Moving to 2D, we consider a quantum well grown in the [001] direction. In general, an asymmetric quantum well introduces an electric field due to the spatial variation of the band edge and gives rise to an additional spin-orbit interaction due to structural inversion asymmetry. This is described by the Bychkov-Rashba Hamiltonian [15], which introduces an energy splitting linear in $k$. For present purposes, however, we shall confine our attention to symmetric quantum wells so that we may neglect the Bychkov-Rashba spin-orbit interaction.

We assume that the conduction band eigenstates are of the form

$$
\left|\psi_{n, \mathbf{k} \pm}\right\rangle=e^{\mathrm{i} \mathbf{k} \cdot \mathbf{r}} \phi_{n}(z)\left|u_{\mathbf{k}}^{ \pm}\right\rangle,
$$

where $\mathbf{k}$ is now the in-plane wavevector and $\phi_{n}(z)$ is an envelope function for the $n$th subband of the well. We should pause to note here that the choice of Cartesian coordinates 


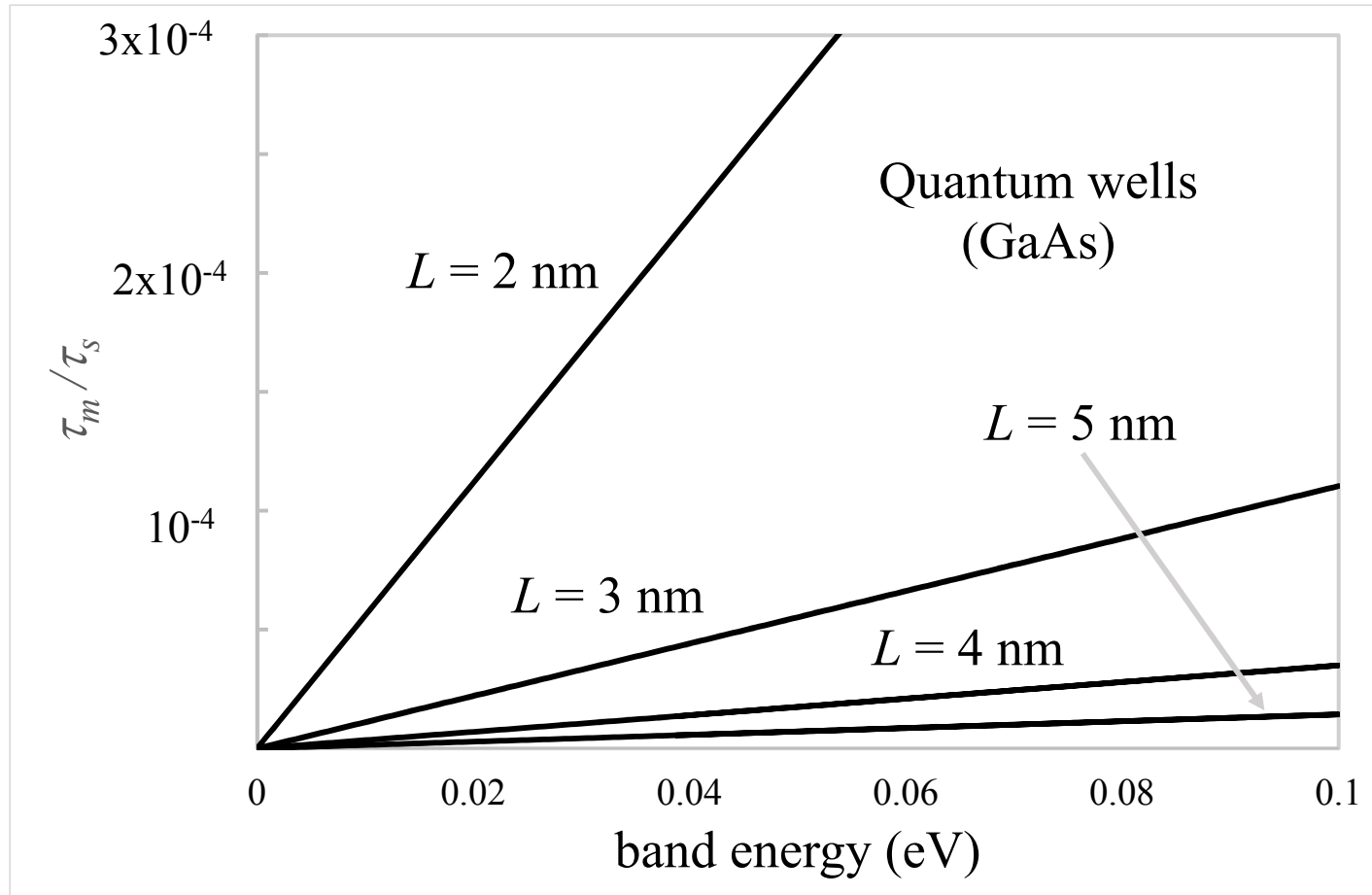

Figure 3. The squared moduli of the Dresselhaus coupling factor $\tau_{m} / \tau_{s}=$ $\left|\left\langle\phi_{\mathbf{k}^{\prime} \downarrow} \mid \phi_{\mathbf{k} \uparrow}\right\rangle\right|^{2}$ in infinite symmetric GaAs quantum wells. $L$ is the well width.

is no longer arbitrary, since the form given earlier for $\boldsymbol{\kappa}$ assumes that $x, y$ and $z$ label crystallographic axes.

In order to obtain tractable, analytical results, we have made certain simplifying assumptions. Firstly, we assume that each bulk band is associated with the same envelope function. Secondly, we make no attempt to properly match the boundary conditions on the $\phi_{n}(z)$. This is only technically justified if we assume that we have an infinite square well. Since we are confining ourselves to consideration of the lowest subbands in the conduction band, we shall take this to be a reasonable approximation.

We may now modify (15) and (17) to read

$$
\left|u_{n, \mathbf{k}}^{ \pm}\right\rangle \equiv \phi_{n}(z)\left|u_{\mathbf{k}}^{ \pm}\right\rangle=U_{n, \mathbf{k}+\partial_{z}}^{ \pm}\left|\chi_{\mathbf{k}}^{ \pm}\right\rangle
$$

where $U_{n, \mathbf{k}+\partial_{z}}^{ \pm}$is

$$
U_{n, \mathbf{k}+\partial_{z}}^{ \pm}=U_{\mathbf{k}+\partial_{z}}^{ \pm} \phi_{n}(z)
$$

in which every instance of $k_{z}$ is replaced by $-\mathrm{i} \partial / \partial z$. Following the same assumptions as above, we put $\Gamma_{\mathbf{k}}^{ \pm} \approx 1$ and write a spin-up state $\left|\phi_{n, \mathbf{k} \uparrow}\right\rangle$ as a superposition of eignenstates.

With these modifications, (35) for the ZSC component of the coupling factor now becomes

$$
\left\langle\phi_{m, \mathbf{k}^{\prime} \downarrow} \mid \phi_{n, \mathbf{k} \uparrow}\right\rangle=-\mathrm{i} \frac{\epsilon_{k}}{\epsilon_{G}^{*}}\left[\frac{k^{\prime}}{k^{2}} e^{i \varphi^{\prime}}\left\langle\phi_{m} \mid \partial_{z} \phi_{n}\right\rangle\right.
$$




$$
\left.+\left\langle\partial_{z} \phi_{m} \mid \phi_{n}\right\rangle \frac{k}{k^{2}} e^{\mathrm{i} \varphi}\right],
$$

where $\partial_{z} \equiv \partial / \partial z$. Since taking the derivative of $\phi_{n}(z)$ changes its parity, the integrals over the $z$ direction disappear for $m=n$. Hence, there is no intra-subband spin relaxation due to this component of coupling factor. We will still have inter subband spin relaxation, with selection rules depending on the exact form of the envelope functions. For an infinite square well, this will be when the difference in subband index is an odd number. This means that even a second order process cannot flip the spin, since an allowed spin flipping transition to another subband must be followed by a return transition, flipping the spin back to its original state.

On the other hand, in 2D, the Dresselhaus component of the coupling factor now becomes linear in $\mathbf{k}$. Neglecting remaining terms proportional to $k^{3}$, the coupling factor is

$$
\begin{aligned}
\left\langle\phi_{m, \mathbf{k}^{\prime} \downarrow} \mid \phi_{n, \mathbf{k} \uparrow}\right\rangle & =\gamma_{D} \frac{\left(\Delta+2 \epsilon_{G}\right)}{\epsilon_{G}\left(\epsilon_{G}+\Delta\right)}\left\langle\partial_{z} \phi_{m} \mid \partial_{z} \phi_{n}\right\rangle \\
& \times\left(\frac{k^{\prime} e^{\mathrm{i} \varphi^{\prime}}+k e^{\mathrm{i} \varphi}}{2}\right) .
\end{aligned}
$$

For intra-subband spin relaxation $m=n$ and in an infinite square well of width $L$, $\left\langle\partial_{z} \phi_{n} \mid \partial_{z} \phi_{n}\right\rangle=k_{n}^{2}=(n \pi / L)^{2}$. Hence we may expect the Dresselhaus contribution to become more important the narrower the well. Again, we note that we still have a finite component to the spin relaxation rate at $\mathbf{k}^{\prime}=\mathbf{k}$. We further note that this linear part of the coupling has rotational symmetry in the plane of the well, unlike the bulk result, which disappears when $\mathbf{k}$ lies along a crystallographic axis.

To quantify the size of this Dresselhaus coupling term, let us assume an infinite quantum well and consider the case of $\mathbf{k}^{\prime}=\mathbf{k}$. We then have

$$
\left|\left\langle\phi_{n, \mathbf{k} \downarrow} \mid \phi_{n, \mathbf{k} \uparrow}\right\rangle\right|^{2}=\left(\gamma_{D} \frac{\left(\Delta+2 \epsilon_{G}\right)}{\epsilon_{G}\left(\epsilon_{G}+\Delta\right)}\right)^{2}\left(\frac{n \pi}{L}\right)^{4} k^{2},
$$

for which there is no angular dependence at all. This is plotted in Fig. 3 for a selection of quantum well widths from 2 to $5 \mathrm{~nm}$. We see that, around the thermal energy, the size of Dresselhaus coupling in 2D is comparable to the bulk value of the ZSC coupling for a well width of $4 \mathrm{~nm}$ and exceeds it for narrower wells. In 2D, room temperature electron mobilities may be of the order 0.1 to $1 \times 10^{4} \mathrm{~cm}^{2} / \mathrm{Vs}$ [16] for well widths around 1 to $5 \mathrm{~nm}$, corresponding to momentum times in the range 0.04 to $0.4 \mathrm{ps}$. So, in a $2 \mathrm{~nm}$ well, for instance, a momentum relaxation time of 0.04 ps would give a spin relaxation time of $180 \mathrm{ps}$ at $\epsilon_{k}=0.04 \mathrm{eV}$, comparable to observed values [9-11]. On the other hand, a $6 \mathrm{~nm}$ well with $\tau_{m}=0.4$ ps would give $\tau_{s}=150 \mathrm{~ns}$, so the contribution is only significant for the narrowest wells. 
The $(n \pi / L)^{4}$ (or $k_{n}^{4}$ ) dependence means that the squared modulus of the coupling factor is proportional to $\epsilon_{n}^{2}$, i.e. the square of the confinement energy of the well. In the absence of any well width dependence on the scattering potential, from (5) we have $1 / \tau_{s}(\epsilon) \propto \epsilon_{n}^{2}$, as found for the spin relaxation time associated with the D'yakonov-Perel mechanism [17]. Whilst this latter process is usually expected to dominate, our result means that contributions from the Elliot process add with the same energy dependence. Note that this result is in contrast to the result of Tackeuchi et al [10], who derive a result of $1 / \tau_{s}(\epsilon) \propto \epsilon_{n}$ using the ZSC model in 2D. Inspecting (43) we see that this would indeed be the energy dependence if it were not for the disappearance of intra-valley spin relaxation for this term as discussed above.

\section{Discussion and conclusions}

The coupling factors derived in Sections 3.3 and 3.4 have been used in conjunction with (8) to compare spin relaxation times to momentum relaxation times. The purpose of this has been to show that the modification of the Elliot process to incorporate the Dresselhaus spin-orbit interaction is important for quantum wells and lower dimensional structures. However, the derivation of (8) is for elastic processes only and involved assuming an isotropic scattering potential. Whilst these assumptions are reasonable for deformation potential acoustic phonon scattering, (8) does not suffice for a quantitative calculation of the spin relaxation in the general case.

For a proper treatment of the spin dynamics, we would need to use our results for the coupling factors in conjunction with a generalization of (4) (to allow for inelastic scattering) in a full dynamical model. This is not a trivial task, especially for the proper treatment of polar-optical phonon (POP) scattering. Our results constitute 'intrinsic' scattering rates, which should be incorporated into a more general spin-density formalism. Such a semi-classical treatment of spin relaxation using a 'ladder method' for dealing with POP scattering in cubic III-V semiconductors has been carried out by Dyson and Ridley in bulk [18] and low-dimensional semiconductors [19]. However, these authors did not adapt the ZSC model for the Elliot process in the latter case. Other dynamical models requiring the expressions derived here as input might include the kinetic spin Bloch equation approach developed by Wu et al (see Ref [20] and further references therein) or Monte Carlo simulations (e.g. Refs [21,22]).

Comparisons to experiment require the calculation of all spin relaxation mechanisms as described above. However, we noted earlier that, with no significant well-width dependence on the scattering potential, the spin relaxation time found here for the Elliot process should have the same dependence on quantum well confinement energy as that for the D'yakonov-Perel mechanism. This $1 / \tau_{s}(\epsilon) \propto \epsilon_{n}^{2}$ dependence is consistent with experimentally observations $[9,11]$ of spin relaxation in quantum wells.

In conclusion, we have considered the Elliot process for spin relaxation with the

inclusion of the Dresselhaus spin-splitting of the conduction band. Consideration of this splitting is essential for any discussion of spin dynamics in materials lacking an inversion 
symmetry, since it acts as an effective magnetic field in which the spin precesses. This leads to a spin relaxation via the D'yakonov-Perel mechanism, belonging to the class of motional narrowing spin relaxation mechanisms.

We have included this aspect of the spin orbit coupling consistently into the description of the admixture spin-eigenstates of the conduction band, which allows for the Elliot process of spin relaxation. We have shown that, to very good approximation, this remains consistent with the existing ZSC model (due to Zawadzki and Szymańska [4] and Chazalviel [5]) in bulk material, although now putting the physics of this process on the same footing as the D'yakonov-Perel mechanism. In this model, the coupling factor between opposite spin components in the initial and final electronic states disappears when the final wavevector $\mathbf{k}^{\prime}$ equals the initial wavevector $\mathbf{k}$.

In symmetric quantum wells, we have found that intra-subband spin relaxation disappears in the ZSC model. The Dresselhaus coupling, on the other hand, remains, becoming increasingly significant as the well becomes narrower. In GaAs wells, the Dresselhaus coupling is comparable to the bulk ZSC coupling for a well width of around $4 \mathrm{~nm}$. We conclude that for analysis of the spin dynamics of quantum wells (and lower dimensional semiconductor structures), this modified version of the Elliot process should be used.

\section{Acknowledgments}

This work is dedicated to the life and work of Naci Balkan, who was an original coinvestigator on the Spin Space project grant. This work is supported by the Engineering and Physical Sciences Research Council.

\section{Appendix A. Theoretical background}

\section{Appendix A.1. The spin-orbit interaction}

The spin-orbit interaction Hamiltonian arising through the coupling of the electron spin $\mathbf{S}$ and orbital angular momentum $\mathbf{L}$ is given by

$$
H_{S O}=\frac{\hbar}{4 m_{0}^{2} c^{2}}(\nabla V \times \mathbf{p}) \cdot \boldsymbol{\sigma}
$$

where $m_{0}$ is the free electron mass, $V$ is the potential energy of the local electric field seen by the electron, $\mathbf{p}$ is the momentum of the electron and $\sigma$ is the Pauli pseudovector taking the Pauli matrices $\sigma_{i}$ as components. On combining the $p$-type valence band states $|X\rangle,|Y\rangle$ and $|Z\rangle$ with spinors $|\uparrow\rangle$ and $|\downarrow\rangle$, we find that the basis-states that diagonalize (A.1) are eigenvectors of the total angular momentum $\mathbf{J}$ (see (B.9) in Appendix B). At $\mathbf{k}=0$, the degeneracy of these states is split by the energy [12]

$$
\Delta=\frac{3 \mathrm{i} \hbar}{4 m_{0}^{2} c^{2}}\left\langle X\left|\frac{\partial V}{\partial x} p_{y}-\frac{\partial V}{\partial y} p_{x}\right| Y\right\rangle .
$$




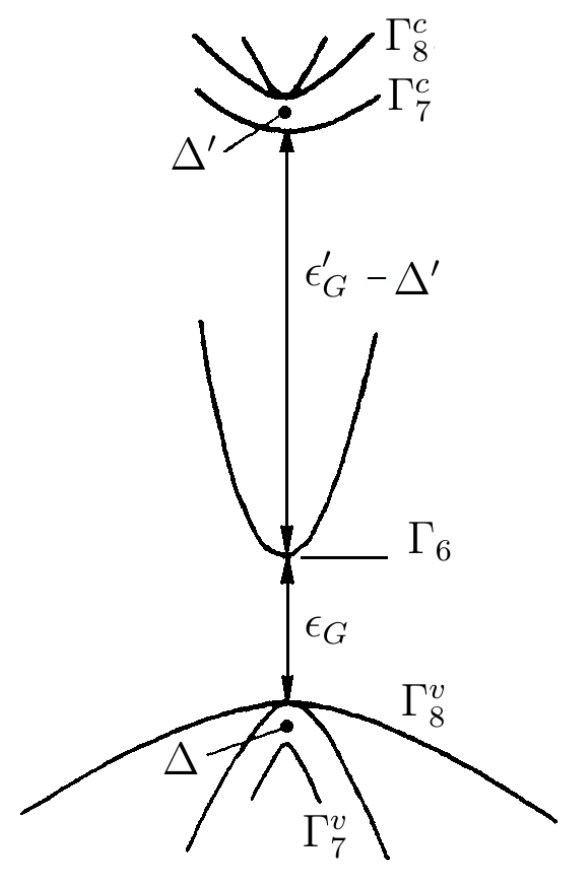

Figure A1. Energy band diagram for a III-V semiconductor.

The higher four states are the heavy and light hole bands, having $j=3 / 2$ and have the symmetry of the double group representation $\Gamma_{8}^{v}$. The lower band is the spin orbit split-off band with $j=1 / 2$ and has the symmetry of $\Gamma_{7}^{v}$ double group representation(see Fig A1).

On combining the $s$-type $\Gamma_{1}^{c}$ conduction band state $|S\rangle$ with spin, we have spinstates with $j=1 / 2$ and $\Gamma_{6}^{c}$ symmetry. These states have zero orbital angular momentum $(l=0)$ and at $\mathbf{k}=0$ are pure spin states. However, at finite $\mathbf{k}$, the conduction band becomes coupled to the valence band states via the $\mathbf{k} \cdot \mathbf{p}$ interaction, composed of matrix elements such as

$$
\frac{\hbar}{m_{0}}\langle S|\mathbf{k} \cdot \mathbf{p}| Z\rangle=\mathrm{i} k_{z} P
$$

This equation conveniently defines the Kane parameter $P$. The actual interaction matrix elements will be between the conduction band states and eigenstates of $\mathbf{J}$ (see Appendix $\mathrm{B}$ for details). This then leads to the conduction band state becoming a k-dependent admixture of spin states.

\section{Appendix A.2. Conduction band spin-splitting}

The Dresselhaus splitting of the conduction band emerges through the interaction with the higher $\Gamma_{7}^{c}$ and $\Gamma_{8}^{c}$ conduction bands (see Fig. A1). We may incorporate the interaction with higher lying states into an 8-band model via Löwdin renormalization [23]. Accordingly, the matrix elements of the 8-band model are extended to second order 
via the prescription

$$
H_{i j} \rightarrow H_{i j}+\sum_{l} \frac{H_{i l} H_{l j}}{\epsilon-H_{l l}},
$$

where the sum is over remote bands. The $\Gamma_{7}^{c}$ and $\Gamma_{8}^{c}$ states, which we shall denote by $\left|X_{l}^{c}\right\rangle$, transform in the same way as a state of the form $\left|X_{l}^{c}\right\rangle \sim a|X\rangle+b|Y\rangle+c|Z\rangle$, so that the matrix element of the $\mathbf{k} \cdot \mathbf{p}$ perturbation becomes

$$
\begin{aligned}
\frac{\hbar}{m_{0}}\langle\mathrm{i} S|\mathbf{k} \cdot \mathbf{p}| Z\rangle & =-\mathrm{i} \frac{\hbar}{m_{0}} k_{z}\left\langle S\left|p_{z}\right| Z\right\rangle \\
& -\mathrm{i} \frac{\hbar}{m_{0}} k_{x} k_{y} \\
& \times \sum_{l} \frac{\left\langle S\left|p_{x}\right| X_{l}^{c}\right\rangle\left\langle X_{l}^{c}\left|p_{y}\right| Z\right\rangle}{\epsilon-H_{l l}},
\end{aligned}
$$

with cyclic permutations of the indices $x, y, z$. Note that combination of $p_{x}$ with the $x$ component of $\left|X_{l}^{c}\right\rangle$ has even parity, as does the combination of the $y$ and $z$ components of $\left|X_{l}^{c}\right\rangle$ with $p_{y}$ and $|Z\rangle$. Hence these matrix element combinations do not disappear.

We now follow the general notation of Refs $[24,25]$ by denoting the second term via

$$
\frac{\hbar^{2}}{m_{c v}} \equiv \frac{\hbar}{m_{0}} \sum_{l} \frac{\left\langle S\left|p_{x}\right| X_{l}^{c}\right\rangle\left\langle X_{l}^{c}\left|p_{y}\right| Z\right\rangle}{\epsilon-H_{l l}} .
$$

Note, however, that we have introduced the factor of $\hbar^{2}$ so that $m_{c v}$ may have dimensions of mass. Using Kane's $P$ parameter, we may then write (A.5) as

$$
\frac{\hbar}{m_{0}}\langle\mathrm{i} S|\mathbf{k} \cdot \mathbf{p}| Z\rangle=P k_{z}-\frac{\mathrm{i} \hbar^{2}}{m_{c v}} k_{x} k_{y} \equiv K_{z}
$$

with its cyclic permutations.

Diagonalizing the renormalized matrix (see Appendix B), we obtain the conduction band energy

$$
\epsilon_{C}(\mathbf{k})=\epsilon_{C}+\frac{\hbar^{2} k^{2}}{2 m^{*}} \pm \gamma_{D}\left(\kappa_{x}^{2}+\kappa_{y}^{2}+\kappa_{z}^{2}\right)^{1 / 2}
$$

where $\epsilon_{C}$ is the conduction band-edge energy, $m^{*}$ is the effective mass (given by (B.26)), $\gamma_{D}$ is the Dresselhaus coefficient

$$
\gamma_{D}=\frac{2}{3} \frac{\hbar^{2} P}{m_{c v}}\left(\frac{\eta}{\epsilon_{G}}\right)
$$

$\epsilon_{G}$ is the energy gap, $\kappa_{z}=k_{z}\left(k_{x}^{2}-k_{y}^{2}\right)$ with cyclic permutations and we have defined

$$
\eta \equiv \frac{\Delta}{\epsilon_{G}+\Delta}
$$

Substituting for $P$ from (B.26) in Appendix B, (A.9) may be re-written in as

$$
\gamma_{D}=\frac{2 \hbar^{3}}{3} \frac{\eta}{m_{c v}}\left(\frac{1}{m^{*}}-\frac{1}{m_{0}}\right)^{1 / 2}\left[2 \epsilon_{G}\left(1-\frac{\eta}{3}\right)\right]^{-1 / 2} .
$$

Note that this expression is usually further approximated by putting $\left(1 / m^{*}-1 / m_{0}\right) \rightarrow$ $1 / m^{*}$ as, for instance, in Ref. [24]. In our calculations we shall use the expression above. 


\section{Appendix B. The cubic Dresselhaus spin splitting}

Following Dresselhaus [2] or Kane [12], we may construct an 8-band $\mathbf{k} \cdot \mathbf{p}$ model for the Hamiltonian

$$
H=H_{0}+H_{S O}
$$

where

$$
H_{0}=\frac{p^{2}}{2 m_{0}}+V(\mathbf{r})
$$

and $H_{S O}$ is given by (A.1) for the spin-orbit coupling term. Writing the Schrödinger equation in terms of Bloch functions, we may cancel out the $e^{\mathrm{ik} \cdot \mathbf{r}}$ factor in the usual fashion to obtain

$$
\begin{aligned}
& {\left[\frac{p^{2}}{2 m_{0}}+V(\mathbf{r})+\frac{\hbar}{4 m_{0}^{2} c^{2}}(\nabla V \times \mathbf{p}) \cdot \boldsymbol{\sigma}+\right.} \\
& \left.+\frac{\hbar}{m_{0}} \mathbf{k} \cdot \mathbf{p}+\frac{\hbar^{2}}{4 m_{0}^{2} c^{2}}(\nabla V \times \mathbf{k}) \cdot \boldsymbol{\sigma}\right] u_{n, \mathbf{k}}= \\
& \left(\epsilon_{n, \mathbf{k}}-\frac{\hbar^{2} k^{2}}{2 m}\right) u_{n, \mathbf{k}} .
\end{aligned}
$$

The fifth term in the square brackets is a $\mathbf{k}$-dependent component of the spin-orbit coupling and is found to be very weak and so generally neglected, as we shall do here.

We employ orbital angular momentum basis states $|S\rangle$ for the $s$-type $(l=0)$ conduction band together with

$$
\begin{aligned}
& \left|R_{+}\right\rangle=\frac{1}{\sqrt{2}}(|X\rangle+i|Y\rangle) \quad\left(l=1, m_{l}=1\right), \\
& \left|R_{-}\right\rangle=\frac{1}{\sqrt{2}}(|X\rangle-i|Y\rangle) \quad\left(l=1, m_{l}=-1\right)
\end{aligned}
$$

and $|Z\rangle\left(l=1, m_{l}=0\right)$ for the $p$-type valence bands. We may then combine these states with spinors via tensor products $|S \uparrow\rangle \equiv|S\rangle \otimes|\uparrow\rangle$ etc. The parameters of the model are then defined by

$$
P=-i \frac{\hbar}{m}\left\langle S\left|p_{i}\right| X_{i}\right\rangle
$$

and

$$
\Delta=\frac{3 i \hbar}{4 m_{0}^{2} c^{2}}\left\langle X_{i}\left|\frac{\partial V}{\partial x_{i}} p_{j}-\frac{\partial V}{\partial x_{j}} p_{i}\right| X_{j}\right\rangle
$$

The following results may then be found fairly easily: 


$$
\begin{aligned}
\left\langle R_{ \pm}\left|H_{S O}\right| R_{ \pm}\right\rangle & = \pm \frac{\Delta}{3} \sigma_{z}, \\
\left\langle R_{ \pm}\left|H_{S O}\right| R_{\mp}\right\rangle & =0, \\
\left\langle R_{ \pm}\left|H_{S O}\right| Z\right\rangle & =\mp \frac{\sqrt{2} \Delta}{3} \sigma_{\mp} \text { and } \\
\left\langle Z\left|H_{S O}\right| R_{ \pm}\right\rangle & =\mp \frac{\sqrt{2} \Delta}{3} \sigma_{ \pm},
\end{aligned}
$$

where

$$
\sigma_{ \pm}=\frac{1}{2}\left(\sigma_{x} \pm i \sigma_{y}\right) .
$$

An $8 \times 8$ matrix is then constructed using basis functions

$$
\begin{aligned}
&\left|u_{1}\right\rangle=\left|S \frac{1}{2}, \frac{1}{2}\right\rangle=|i S \uparrow\rangle, \\
&\left|u_{2}\right\rangle=\left|S \frac{1}{2},-\frac{1}{2}\right\rangle=|i S \downarrow\rangle, \\
&\left|u_{3}\right\rangle=\left|\frac{3}{2}, \frac{3}{2}\right\rangle=\left|R_{+} \uparrow\right\rangle, \\
&\left|u_{4}\right\rangle=\left|\frac{3}{2}, \frac{1}{2}\right\rangle=-\sqrt{\frac{1}{3}}\left|R_{+} \downarrow\right\rangle+\sqrt{\frac{2}{3}}|Z \uparrow\rangle, \\
&\left|u_{5}\right\rangle=\left|\frac{3}{2},-\frac{1}{2}\right\rangle=\sqrt{\frac{1}{3}}\left|R_{-} \uparrow\right\rangle+\sqrt{\frac{2}{3}}|Z \downarrow\rangle, \\
&\left|u_{6}\right\rangle=\left|\frac{3}{2},-\frac{3}{2}\right\rangle=\left|R_{-} \downarrow\right\rangle, \\
&\left|u_{7}\right\rangle=\left|\frac{1}{2}, \frac{1}{2}\right\rangle=\sqrt{\frac{2}{3}}\left|R_{+} \downarrow\right\rangle+\sqrt{\frac{1}{3}}|Z \uparrow\rangle, \\
&\left|u_{8}\right\rangle=\left|\frac{1}{2},-\frac{1}{2}\right\rangle=\sqrt{\frac{2}{3}}\left|R_{-} \uparrow\right\rangle-\sqrt{\frac{1}{3}}|Z \downarrow\rangle,
\end{aligned}
$$

for which the valence band states can be shown to be eigenvectors of the spin-orbit energy $H_{S O}$ and hence (in the absence of additional strain terms) diagonalize the matrix for $\mathbf{k}=0$.

To find the energy eigenvalues $\epsilon$ for non-zero $\mathbf{k}$, we can use the general result

$$
\left|\begin{array}{cc}
A & B \\
B^{\dagger} & C
\end{array}\right|=|C|\left|A-B C^{-1} B^{\dagger}\right|=0,
$$

where, in our case, we have

$$
\begin{aligned}
& A=\left[\begin{array}{cc}
\Delta \epsilon_{C} & 0 \\
0 & \Delta \epsilon_{C}
\end{array}\right], \\
& \Delta \epsilon_{C}=\epsilon_{C}+\frac{\hbar^{2} k^{2}}{2 m_{0}}-\epsilon,
\end{aligned}
$$


$\epsilon_{C}$ is the conduction band edge energy and $m_{0}$ is the free electron mass. The matrix $B$ may be written in the general form

$$
B=\left[\begin{array}{llllll}
V_{13} & V_{14} & V_{15} & V_{16} & V_{17} & V_{18} \\
V_{23} & V_{24} & V_{25} & V_{26} & V_{27} & V_{28}
\end{array}\right],
$$

where $V_{1 i}=\left\langle i S \uparrow|H| u_{i}\right\rangle$ and $V_{2 i}=\left\langle i S \downarrow|H| u_{i}\right\rangle$. In the absence of additional terms due to strain or the interaction with other remote bands, the matrix $C$ is

$$
C=\left[\begin{array}{cccccc}
\Delta \epsilon_{3} & 0 & 0 & 0 & 0 & 0 \\
0 & \Delta \epsilon_{4} & 0 & 0 & 0 & 0 \\
0 & 0 & \Delta \epsilon_{5} & 0 & 0 & 0 \\
0 & 0 & 0 & \Delta \epsilon_{6} & 0 & 0 \\
0 & 0 & 0 & 0 & \Delta \epsilon_{7} & 0 \\
0 & 0 & 0 & 0 & 0 & \Delta \epsilon_{8}
\end{array}\right]
$$

where the $\Delta \epsilon_{i}$ for the valence band edges are defined analogously to (B.12). The determinant of this is simply

$$
|C|=\prod_{i=3} \Delta \epsilon_{i}=\Delta \epsilon_{V}^{4} \epsilon_{S O}^{2} .
$$

For the conduction band $|C| \neq 0$ (this will remain true even in the general case where $C$ has non-zero off-diagonal elements), so we may divide (B.10) by $|C|$ to give the characteristic equation for a two band model

$$
\left|A-B C^{-1} B^{\dagger}\right|=0 \text {. }
$$

From (B.13), we have

$$
B C^{-1} B^{\dagger}=\sum_{i=3} \frac{1}{\Delta \epsilon_{i}}\left[\begin{array}{cc}
\left|V_{1 i}\right|^{2} & V_{1 i} V_{2 i}^{*} \\
V_{1 i}^{*} V_{2 i} & \left|V_{2 i}\right|^{2}
\end{array}\right]
$$

and hence (B.16) becomes

$$
\left|\begin{array}{cc}
\Delta \epsilon_{C}-\sum\left|V_{1 i}\right|^{2} / \Delta \epsilon_{i} & -\sum V_{1 i} V_{2 i}^{*} / \Delta \epsilon_{i} \\
-\sum V_{1 i}^{*} V_{2 i} / \Delta \epsilon_{i} & \Delta \epsilon_{C}-\sum\left|V_{2 i}\right|^{2} / \Delta \epsilon_{i}
\end{array}\right|=0 .
$$

We then have the solution

$$
\begin{aligned}
\Delta \epsilon_{C} & =\frac{1}{2} \sum_{i} \frac{\left|V_{1 i}\right|^{2}+\left|V_{2 i}\right|^{2}}{\Delta \epsilon_{i}} \\
& \pm \frac{1}{2}\left[\left(\sum_{i} \frac{\left|V_{1 i}\right|^{2}-\left|V_{2 i}\right|^{2}}{\Delta \epsilon_{i}}\right)^{2}\right. \\
& \left.+4\left|\sum_{i} \frac{V_{1 i} V_{2 i}^{*}}{\Delta \epsilon_{i}}\right|^{2}\right]^{1 / 2} \equiv \Delta \epsilon_{C 0} \pm \epsilon_{D}(\mathbf{k}) .
\end{aligned}
$$


Note that both the right and left hand sides of (B.19) involve $\epsilon$, so we cannot obtain an explicit expression for the energy from this. However, for small values of $k$, we may make the approximation $\epsilon \sim \epsilon_{C}$ to simplify the right-hand-side as we need to.

We may incorporate the interaction with other bands via Löwdin perturbation theory as described earlier in Appendix A.2. Using the result of (A.7), we may then construct an explicit expression for the $B$ matrix

$$
\begin{aligned}
& B=\left[\begin{array}{ccc}
K_{+} & \sqrt{2 / 3} K_{z} & \sqrt{1 / 3} K_{-} \\
0 & -\sqrt{1 / 3} K_{+} & \sqrt{2 / 3} K_{z}
\end{array} \ldots\right. \\
& \left.\begin{array}{cccc} 
& 0 & \sqrt{1 / 3} K_{z} & \sqrt{2 / 3} K_{-} \\
& K_{-} & \sqrt{2 / 3} K_{+} & -\sqrt{1 / 3} K_{z}
\end{array}\right]
\end{aligned}
$$

where

$$
K_{ \pm}=\frac{1}{\sqrt{2}}\left(K_{x} \pm i K_{y}\right)
$$

It will also be useful to define the analogous expression for $k$ vector

$$
k_{ \pm}=\frac{1}{\sqrt{2}}\left(k_{x} \pm i k_{y}\right)
$$

We then obtain the intermediate results

$$
\left|K_{ \pm}\right|^{2}=\left(P^{2}+\frac{\hbar^{4} k_{z}^{2}}{m_{c v}^{2}}\right)\left|k_{ \pm}\right|^{2} \pm \frac{\hbar^{2} P}{m_{c v}} k_{z}\left(k_{x}^{2}-k_{y}^{2}\right) \text {. }
$$

and

$$
\left|K_{z}\right|^{2}=P^{2} k_{z}^{2}+\frac{\hbar^{4} k_{x}^{2} k_{y}^{2}}{m_{c v}^{2}}
$$

To begin with, let us calculate the first term in (B.19)

$$
\begin{aligned}
& \frac{1}{2} \sum_{i} \frac{\left|V_{1 i}\right|^{2}+\left|V_{2 i}\right|^{2}}{\Delta \epsilon_{i}} \\
& =\frac{1}{3}\left(\left|K_{+}\right|^{2}+\left|K_{-}\right|^{2}+\left|K_{z}\right|^{2}\right)\left(\frac{2}{\Delta \epsilon_{V}}+\frac{1}{\Delta \epsilon_{S O}}\right), \\
& =\frac{1}{3}\left(P^{2} k^{2}+\frac{\hbar^{4}}{m_{c v}^{2}}\left[k_{x}^{2} k_{y}^{2}+k_{x}^{2} k_{y}^{2}+k_{x}^{2} k_{y}^{2}\right]\right) \\
& \times\left(\frac{2}{\Delta \epsilon_{V}}+\frac{1}{\Delta \epsilon_{S O}}\right) .
\end{aligned}
$$

Now $\Delta \epsilon_{S O}=\Delta \epsilon_{V}-\Delta$, so putting $\Delta \epsilon_{V} \approx \epsilon_{V}-\epsilon_{C}=-\epsilon_{G}$, the energy gap, we may rearrange (B.19) using (B.12) and neglecting the $k^{4}$ terms to give

$$
\epsilon_{C}(\mathbf{k})=\epsilon_{C}+\frac{\hbar^{2} k^{2}}{2 m^{*}} \pm \epsilon_{D}(\mathbf{k})
$$


where the effective mass $m^{*}$ is defined via

$$
\frac{1}{m^{*}}=\frac{1}{m_{0}}+\frac{2 P^{2}}{3 \hbar^{2}}\left(\frac{2}{\epsilon_{G}}+\frac{1}{\epsilon_{G}+\Delta}\right) .
$$

Approaching the evaluation of $\epsilon_{D}(\mathbf{k})$ in manageable steps, we have

$$
\begin{aligned}
\sum_{i} \frac{\left|V_{1 i}\right|^{2}-\left|V_{2 i}\right|^{2}}{\Delta \epsilon_{i}} & =\frac{2}{3}\left(\left|K_{+}\right|^{2}-\left|K_{-}\right|^{2}\right) \\
& \times\left(\frac{1}{\Delta \epsilon_{V}}-\frac{1}{\Delta \epsilon_{S O}}\right) .
\end{aligned}
$$

Using (B.23) and (A.10) with $\Delta \epsilon_{S O}=\Delta \epsilon_{V}-\Delta$ and $\Delta \epsilon_{V} \approx \epsilon_{V}-\epsilon_{C}=-\epsilon_{G}$ again,

$$
\left(\sum_{i} \frac{\left|V_{1 i}\right|^{2}-\left|V_{2 i}\right|^{2}}{\Delta \epsilon_{i}}\right)^{2}=\frac{16}{9} \frac{\hbar^{4} P^{2}}{m_{c v}^{2}} k_{z}^{2}\left(k_{x}^{2}-k_{y}^{2}\right)^{2}\left(\frac{\eta}{\epsilon_{G}}\right)^{2} .
$$

Meanwhile,

$$
\begin{aligned}
\sum_{i} \frac{V_{1 i} V_{2 i}^{*}}{\Delta \epsilon_{i}} & =-\frac{\sqrt{2}}{3}\left(K_{z} K_{+}^{*}-K_{-} K_{z}^{*}\right) \\
& \times\left(\frac{1}{\Delta \epsilon_{V}}-\frac{1}{\Delta \epsilon_{S O}}\right)
\end{aligned}
$$

where

$$
\begin{aligned}
K_{z} K_{+}^{*}-K_{-} K_{z}^{*} & =-\frac{\sqrt{2} \hbar^{2} P}{m_{c v}}\left[k_{x}\left(k_{y}^{2}-k_{z}^{2}\right)\right. \\
& \left.-i k_{y}\left(k_{z}^{2}-k_{x}^{2}\right)\right]
\end{aligned}
$$

which gives, on treating $\Delta \epsilon_{V}$ and $\Delta \epsilon_{S O}$ as before,

$$
\begin{aligned}
4\left|\sum_{i} \frac{V_{1 i} V_{2 i}^{*}}{\Delta \epsilon_{i}}\right|^{2} & =\frac{16}{9} \frac{\hbar^{4} P^{2}}{m_{c v}^{2}}\left[k_{x}^{2}\left(k_{y}^{2}-k_{z}^{2}\right)^{2}\right. \\
& \left.+k_{y}^{2}\left(k_{z}^{2}-k_{x}^{2}\right)^{2}\right]\left(\frac{\eta}{\epsilon_{G}}\right)^{2} .
\end{aligned}
$$

Putting these results together, we have, from (B.19),

$$
\begin{aligned}
\epsilon_{D}(\mathbf{k}) & = \pm \frac{2}{3} \frac{\hbar^{2} P}{m_{c v}}\left(\frac{\eta}{\epsilon_{G}}\right) \\
& \times\left[k_{x}^{2}\left(k_{y}^{2}-k_{z}^{2}\right)^{2}+k_{y}^{2}\left(k_{z}^{2}-k_{x}^{2}\right)^{2}\right. \\
& \left.+k_{z}^{2}\left(k_{x}^{2}-k_{y}^{2}\right)^{2}\right]^{1 / 2}
\end{aligned}
$$


which, using the expression for $\gamma_{D}$ in (A.9), gives us the energy splittings given earlier as the eigenvalues of the matrix $H_{D}$ in (13). Combining with (B.25) for the dispersion relations then gives us (A.8) for the conduction band energy as required.

\section{Appendix C. Admixture spin state eigenvectors}

The eigenvectors of the system may be found in terms of the coefficients of the basis states of (B.9) from the matrix equation of the system. On writing out the matrix in full, we find the relations

$$
\begin{aligned}
& \Delta \epsilon_{C} a_{1}+\sum_{i=3} V_{1 i} a_{i}=0, \\
& \Delta \epsilon_{C} a_{2}+\sum_{i=3} V_{2 i} a_{i}=0,
\end{aligned}
$$

and

$$
a_{i}=-\frac{V_{1 i}^{*} a_{1}+V_{2 i}^{*} a_{2}}{\Delta \epsilon_{i}}
$$

These coefficients may then be fully specified (to within a phase factor) by imposing the normalization condition

$$
\left|a_{1}\right|^{2}+\left|a_{2}\right|^{2}+\sum_{i=3}\left|a_{i}\right|^{2}=1 .
$$

From (C.1) and (C.3)

$$
\Delta \epsilon_{C} a_{1}=\sum_{i=3} \frac{\left|V_{1 i}\right|^{2}}{\Delta \epsilon_{i}} a_{1}+\sum_{i=3} \frac{V_{1 i} V_{2 i}^{*}}{\Delta \epsilon_{i}} a_{2},
$$

So

$$
\left(\Delta \epsilon_{C}-\sum_{i=3} \frac{\left|V_{1 i}\right|^{2}}{\Delta \epsilon_{i}}\right) a_{1}=\sum_{i=3} \frac{V_{1 i} V_{2 i}^{*}}{\Delta \epsilon_{i}} a_{2} .
$$

Neglecting quartic terms, we find

$$
\sum_{i} \frac{\left|V_{1 i}\right|^{2}}{\Delta \epsilon_{i}}=-\left[\frac{\hbar^{2} k^{2}}{2 m^{*}}\left(1-\frac{m^{*}}{m_{0}}\right)+\gamma_{D} \kappa_{z}\right]
$$

and

$$
\sum_{i} \frac{V_{1 i} V_{2 i}^{*}}{\Delta \epsilon_{i}}=-\gamma_{D}\left(\kappa_{x}-i \kappa_{y}\right)
$$

Equation(C.6) is then

$$
\begin{aligned}
& \left(\epsilon_{C}+\frac{\hbar^{2} k^{2}}{2 m^{*}}+\gamma_{D} \kappa_{z}-\epsilon\right) a_{1} \\
& =-\gamma_{D}\left(\kappa_{x}-i \kappa_{y}\right) a_{2} .
\end{aligned}
$$


From (A.8), we had $\epsilon=\epsilon_{C}+\hbar^{2} k^{2} /\left(2 m^{*}\right) \pm \gamma_{D}|\boldsymbol{\kappa}|$, so we have

$$
\left(\kappa_{z} \mp|\kappa|\right) a_{1}^{ \pm}=-\left(\kappa_{x}-i \kappa_{y}\right) a_{2}^{ \pm}
$$

which, representing $\boldsymbol{\kappa}$ in terms of spherical polar coordinates, may be written

$$
( \pm 1-\cos \theta) a_{1}^{ \pm}=\sin \theta e^{-i \phi} a_{2}^{ \pm}
$$

Writing this as

$$
G_{1}^{ \pm} a_{1}^{ \pm}=G_{2} a_{2}^{ \pm}
$$

and using the normalization condition (C.4), we find

$$
\begin{aligned}
\left|a_{1}^{ \pm}\right|^{2} & =\frac{\left|G_{2}\right|^{2}}{\left|G_{1}^{ \pm}\right|^{2}+\left|G_{2}\right|^{2}}\left(1-\sum_{i=3}\left|a_{i}\right|^{2}\right) \\
& =\left|\chi_{1}^{ \pm}\right|^{2}\left(1-\sum_{i=3}\left|a_{i}\right|^{2}\right),
\end{aligned}
$$

and

$$
\begin{aligned}
\left|a_{2}^{ \pm}\right|^{2} & =\frac{\left|G_{1}^{ \pm}\right|^{2}}{\left|G_{1}^{ \pm}\right|^{2}+\left|G_{2}\right|^{2}}\left(1-\sum_{i=3}\left|a_{i}\right|^{2}\right) \\
& =\left|\chi_{2}^{ \pm}\right|^{2}\left(1-\sum_{i=3}\left|a_{i}\right|^{2}\right),
\end{aligned}
$$

where $\chi_{1}^{ \pm}$and $\chi_{2}^{ \pm}$are the spin coefficients of the two-band model of (14). That is,

$$
\left|\chi_{\mathbf{k}}^{ \pm}\right\rangle=\left[\begin{array}{c}
\chi_{1}^{ \pm} \\
\chi_{2}^{ \pm}
\end{array}\right]
$$

From (C.3) and (C.11), we find that

$$
\begin{aligned}
\sum_{i=3}\left|a_{i}\right|^{2} & =\sum_{i=3} \frac{\left|V_{1 i}^{*} G_{2}+V_{2 i}^{*} G_{1}^{ \pm}\right|^{2}}{\left|G_{2}\right|^{2} \Delta \epsilon_{i}^{2}}\left|a_{1}^{ \pm}\right|^{2} \\
& =\sum_{i=3} \frac{\left|V_{1 i}^{*} G_{2}+V_{2 i}^{*} G_{1}^{ \pm}\right|^{2}}{\left|G_{1}^{ \pm}\right|^{2} \Delta \epsilon_{i}^{2}}\left|a_{2}^{ \pm}\right|^{2},
\end{aligned}
$$

allowing us to re-write (C.12) and (C.13) as

$$
\left|a_{1}^{ \pm}\right|^{2}=\left|\chi_{1}^{ \pm}\right|^{2}\left[1+\frac{\Sigma}{\left|G_{1}^{ \pm}\right|^{2}+\left|G_{2}\right|^{2}}\right]^{-1}
$$


and

$$
\left|a_{2}^{ \pm}\right|^{2}=\left|\chi_{2}^{ \pm}\right|^{2}\left[1+\frac{\Sigma}{\left|G_{1}^{ \pm}\right|^{2}+\left|G_{2}\right|^{2}}\right]^{-1}
$$

where

$$
\Sigma=\sum_{i=3} \frac{\left|V_{1 i}^{*} G_{2}+V_{2 i}^{*} G_{1}^{ \pm}\right|^{2}}{\Delta \epsilon_{i}^{2}}
$$

After some algebra, we find that

$$
\left[1+\frac{\Sigma}{\left|G_{1}^{ \pm}\right|^{2}+\left|G_{2}\right|^{2}}\right]^{-1}=a_{0}^{2}\left|\Gamma_{\mathbf{k}}^{ \pm}\right|^{2}
$$

where

$$
a_{0}^{2}=\frac{\epsilon_{G}^{2}\left(\epsilon_{G}+\Delta\right)^{2}}{D^{2}}
$$

$D$ is as given in $(23)$,

$$
\left|\Gamma_{\mathbf{k}}^{ \pm}\right|^{2}=\frac{D^{2}}{D^{2}+U_{\mathbf{k}}^{ \pm}}
$$

and

$$
U_{\mathbf{k}}^{ \pm}=\epsilon_{G}\left(\epsilon_{G}+\Delta\right)\left(2 \epsilon_{G}+\Delta\right) \gamma_{D}\left\langle\chi^{ \pm}|\boldsymbol{\sigma} \cdot \boldsymbol{\kappa}| \chi^{ \pm}\right\rangle .
$$

Noting that $\gamma_{D}\left\langle\chi^{ \pm}|\boldsymbol{\sigma} \cdot \boldsymbol{\kappa}| \chi^{ \pm}\right\rangle$is the energy splitting $\epsilon_{D}$ of the conduction band due to spin, we may rewrite this as $U_{\mathbf{k}}^{ \pm}= \pm V_{\mathbf{k}}$ as given earlier in (25).

The coefficients of the other basis states may then be found from (C.3) using the results above for $a_{1}^{ \pm}$and $a_{2}^{ \pm}$. Having obtained these, it turns out that a convenient formulation of the eigenstates $\left|u_{\mathbf{k}}^{ \pm}\right\rangle$emerges if we consider the coefficients of $|X\rangle,|Y\rangle$ and $|Z\rangle$.

Defining $a, b$ and $c$ as earlier by (20), (21) and (22) respectively, we find

$$
\begin{aligned}
& \left\langle X \mid u_{\mathbf{k}}^{ \pm}\right\rangle=\left[c K_{x}^{*}-i \frac{b}{\sqrt{2}} K_{y}^{*} \sigma_{z}+i \frac{b}{\sqrt{2}} K_{z}^{*} \sigma_{y}\right] \Gamma^{ \pm}\left|\chi^{ \pm}\right\rangle, \\
& \left\langle Y \mid u_{\mathbf{k}}^{ \pm}\right\rangle=\left[c K_{y}^{*}+i \frac{b}{\sqrt{2}} K_{x}^{*} \sigma_{z}-i \frac{b}{\sqrt{2}} K_{z}^{*} \sigma_{x}\right] \Gamma^{ \pm}\left|\chi^{ \pm}\right\rangle
\end{aligned}
$$

and

$$
\left\langle Z \mid u_{\mathbf{k}}^{ \pm}\right\rangle=\left[c K_{z}^{*}-i \frac{b}{\sqrt{2}} K_{x}^{*} \sigma_{y}+i \frac{b}{\sqrt{2}} K_{y}^{*} \sigma_{x}\right] \Gamma^{ \pm}\left|\chi^{ \pm}\right\rangle .
$$

Combing these results, we obtain 


$$
\begin{aligned}
\left|u_{\mathbf{k}}^{ \pm}\right\rangle & =\Gamma_{\mathbf{k}}^{ \pm}\left[a|i S\rangle+c \mathbf{K}^{*} \cdot|\mathbf{R}\rangle+\frac{i b}{\sqrt{2}}\left(\mathbf{K}^{*} \times|\mathbf{R}\rangle\right) \cdot \boldsymbol{\sigma}\right] \\
& \times\left|\chi_{\mathbf{k}}^{ \pm}\right\rangle
\end{aligned}
$$

where the vectors $|\mathbf{R}\rangle$ and $\mathbf{K}$ are defined as earlier in (18) and (19). This may then be written in terms of an operator acting on the spin states as given in (15) and (17).

\section{Appendix D. Coupling factor}

In Section 3.3 it is shown that the coupling factor may be written as

$$
\left\langle\phi_{\mathbf{k}^{\prime} \downarrow} \mid \phi_{\mathbf{k} \uparrow}\right\rangle=\left\langle\downarrow\left|U_{\mathbf{k}^{\prime}}^{\dagger} U_{\mathbf{k}}\right| \uparrow\right\rangle
$$

where the $†$ superscript indicates complex conjugation. Using (17) we then find

$$
\left\langle\phi_{\mathbf{k}^{\prime} \downarrow} \mid \phi_{\mathbf{k} \uparrow}\right\rangle=b\left(\frac{b}{2}-\sqrt{2} c\right) \sqrt{2}\left(K_{+}^{\prime} K_{z}^{*}-K_{z}^{\prime} K_{-}^{*}\right) .
$$

Using (21) and (22), we have

$$
b\left(\frac{b}{2}-\sqrt{2} c\right)=-\frac{\Delta\left(\Delta+2 \epsilon_{G}\right)}{3 D^{2}} .
$$

For small $k$, we may approximate $D^{2} \approx \epsilon_{G}^{2}\left(\epsilon_{G}+\Delta\right)^{2}$. Then, using (B.26) for the effective mass, we find

$$
b\left(\frac{b}{2}-\sqrt{2} c\right) \approx-\frac{\epsilon_{k}}{\epsilon_{G}^{*}} \frac{1}{P^{2} k^{2}} .
$$

where $\epsilon_{k}=\hbar^{2} k^{2} /\left(2 m^{*}\right)$ and

$$
\frac{1}{\epsilon_{G}^{*}}=\left(1-\frac{m^{*}}{m_{0}}\right) \frac{\Delta\left(\Delta+2 \epsilon_{G}\right)}{\epsilon_{G}\left(\epsilon_{G}+\Delta\right)\left(3 \epsilon_{G}+2 \Delta\right)} .
$$

Neglecting terms proportional to $k^{4}$, the $\mathbf{k}$ dependent factor of (D.2) is given by

$$
\begin{aligned}
\sqrt{2}\left(K_{+}^{\prime} K_{z}^{*}-K_{z}^{\prime} K_{-}^{*}\right) & =P^{2}\left(k_{x}^{\prime}+\mathrm{i} k_{y}^{\prime}\right) k_{z} \\
& -P^{2} k_{z}^{\prime}\left(k_{x}+\mathrm{i} k_{y}\right) \\
& -\frac{P \hbar^{2}}{m_{c v}}\left[\left(k_{x}^{\prime}+k_{x}\right)\left(k_{y}^{\prime} k_{y}-k_{z}^{\prime} k_{z}\right)\right. \\
& \left.+\mathrm{i}\left(k_{y}^{\prime}+k_{y}\right)\left(k_{z}^{\prime} k_{z}-k_{x}^{\prime} k_{x}\right)\right] .
\end{aligned}
$$

Usually, we also neglect terms in $k^{3}$, so that (D.2) becomes

$$
\left\langle\phi_{\mathbf{k}^{\prime} \downarrow} \mid \phi_{\mathbf{k} \uparrow}\right\rangle=-\frac{\epsilon_{k}}{\epsilon_{G}^{*}} \frac{k^{\prime}}{k}\left[\sin \vartheta^{\prime} \cos \vartheta e^{\mathrm{i} \varphi^{\prime}}-\sin \vartheta \cos \vartheta^{\prime} e^{\mathrm{i} \varphi}\right],
$$


which is the ZSC model as given earlier.

For $\mathbf{k}^{\prime}=\mathbf{k}$, the ZSC term disappears and, using (D.3) and (D.6) with the small $k$ approximation, (D.2) becomes

$$
\left\langle\phi_{\mathbf{k} \downarrow} \mid \phi_{\mathbf{k} \uparrow}\right\rangle=\frac{\Delta\left(\Delta+2 \epsilon_{G}\right)}{3 \epsilon_{G}^{2}\left(\epsilon_{G}+\Delta\right)^{2}} \frac{2 P \hbar^{2}}{m_{c v}}\left(\kappa_{x}+i \kappa_{y}\right) .
$$

Using (A.9), this may be re-written as

$$
\left\langle\phi_{\mathbf{k} \downarrow} \mid \phi_{\mathbf{k} \uparrow}\right\rangle=\gamma_{D} \frac{\left(\Delta+2 \epsilon_{G}\right)}{\epsilon_{G}\left(\epsilon_{G}+\Delta\right)}\left(\kappa_{x}+\mathrm{i} \kappa_{y}\right) .
$$

\section{References}

[1] Elliott R J 1954 Phys. Rev. 96266

[2] Dresselhaus G 1955 Phys. Rev. 100580

[3] Yafet Y 1963 Solid State Physics 14 1-98

[4] Zawadzki W and Szymańska W 1971 Phys. Status Solidi B 45 415-432

[5] Chazalviel J N 1975 Phys. Rev. B 111555

[6] D'yakonov M I and Perel V I 1972 Sov. Phys. Solid State. 13 3023-3026

[7] Song P H and Kim K W 2002 Phys. Rev. B 66035207

[8] Fishman G and Lampel G 1977 Phys. Rev. B 16820

[9] Tackeuchi A, Nishikawa Y and Wada O 1996 Appl. Phys. Lett 68797

[10] Tackeuchi A, Kuroda T, Nishikawa Y et al. 1999 Japanese journal of applied physics 384680

[11] Malinowski A, Britton R S, Grevatt T, Harley R T, Ritchie D A and Simmons M Y 2000 Phys. Rev. B 6213034

[12] Kane E O 1957 J. Phys. Chem. Solids. 1249

[13] Wang G, Liu B L, Balocchi A, Renucci P, Zhu C R, Amand T, Fontaine C and Marie X 2013 Nat. Commun. 42372

[14] Vurgaftman I, Meyer J R and Ram-Mohan L R 2001 J. Appl. Phys. 895815

[15] Bychkov Y A and Rashba E 1984 Sov. Phys. JETP Lett. 3978

[16] Gottinger R, Gold A, Abstreiter G, Weimann G and Schlapp W 1988 EPL (Europhysics Letters) 6183

[17] D'yakonov M I and Kachorovskii V Y 1986 Sov. Phys. Semicond. 20110

[18] Dyson A and Ridley B K 2004 Phys. Rev. B 69125211

[19] Dyson A and Ridley B K 2005 Phys. Rev. B 72045326

[20] Wu M W, Jiang J H, Weng M Q 2010 Physics Reports 493 61-236

[21] Barry E A, Kiselev A A and Kim K W 2003 Appl. Phys. Lett. 82 3686-3688

[22] Pramanik S, Bandyopadhyay S, Cahay M 2003 Phys. Rev. B 68075313

[23] Löwdin P O 1951 J. Chem. Phys. 19 1396-1401

[24] Pikus G E and Titkov A N 1984 Spin relaxation under optical orientation in semiconductors Optical Orientation vol 8 ed Meier F and Zakharchenya B P (North Holland) chap 3, pp 73131

[25] Pikus G E, Maruschak V A and Titkov A N 1988 Sov. Phys. Semicond. 22115 